\title{
Epilepsia partialis continua present with shoulder joint-trunk-hip joint rhythmic clonic seizure:
}

\section{a case report}

\author{
Yudan Lv \\ Zan Wang \\ Fengna Chu \\ Chang Liu \\ Hongmei Meng \\ Department of Neurology and \\ Neuroscience Center, The First \\ Hospital of Jilin University, ChangChun, \\ People's Republic of China
}

\author{
This article was published in the following Dove Press journal: \\ Neuropsychiatric Disease and Treatment \\ 19 September 2016 \\ Number of times this article has been viewed
}

Correspondence: Hongmei Meng Department of Neurology and

Neuroscience Center, The First Hospital of Jilin University, 7I Xinmin Street, ChangChun, People's Republic of China Tel +86I3654362588

Email hongmeiyp@।26.com

\begin{abstract}
Epilepsia partialis continua (EPC) is a particular type of epilepsy which is distinguished from "common epilepsy" by its characteristic semiological features. However, unusual manifestations should be described in order to give awareness at clinical diagnosis. In this case report we describe a rare EPC case not previously reported, in which left shoulder joint-trunk-hip joint jerk was present for 1 week. Abnormal electroencephalogram and cerebrospinal fluid results supported a diagnosis of viral encephalitis. Antiepileptic treatment seemed effective. Our report emphasizes that a detailed functional anatomical analysis and synchronized electroencephalogram discharge should be done to avoid misdiagnosis in patients with synchronic shoulder-trunk-hip jerk symptoms.
\end{abstract}

Keywords: EPC, shoulder-trunk-hip, EEG, clonic seizure, virus encephalitis

\section{Introduction}

Epilepsia partialis continua (EPC) is a rare form of focal status epilepticus. It may have vascular, immune-mediated, neoplastic, or metabolic toxic causes. The origin of EPC has been linked to the motor cortex. EPC is a particular form of epilepsy that is different from "common epilepsy" because it does not have the tendency to spread. The most frequently used clinical definition ${ }^{1,2}$ describes it as:

EPC is spontaneous regular or irregular clonic muscular twitching affecting a limited part of the body, sometimes aggravated by action or sensory stimuli, occurring for a minimum of 1 hour, and recurring at intervals of no more than 10 seconds.

Characteristic semiological features enable EPC to be diagnosed and distinguished from other movement disorders. Additional electrophysiological studies, such as an electroencephalogram (EEG), can contribute to enhance the degree of certainty. However, in recent years some unusual manifestations have been described: Vukadinovic et al described a case of lingual EPC in 2007, ${ }^{3}$ and Espay et al described a case of hemifacial spasm. ${ }^{4}$ In our case, we report an unusual case of EPC presenting with shoulder jointtrunk-hip joint rhythmic jerk, which has not been reported before, in order to bring awareness for clinicians during clinical diagnosis. Written informed consent was obtained from the patient for publication of this case report and any accompanying images and this case report was approved by the First Hospital of Jilin University's Research Ethics Board.

\section{Case report}

A male Chinese patient, 33-years-old, was admitted to our hospital after unconsciousness and seizure onset. A family member of the patient reported that the patient had 
presented with fever a week before, then gradually fell into unconsciousness during which time the patient experienced three seizures. At admission, the patient's body temperature was over $39^{\circ} \mathrm{C}$, mental symptoms including hallucination and talking nonsense and strange seizures occurred, such as left shoulder-trunk-hip rhythmic jerks, which made diagnosis difficult. The patient's abnormal symptoms occurred continuously and disturbed his normal daily life. Because of his mental symptoms, we could not do a neurological examination. A lumbar puncture was done on the fifth day and the cerebrospinal fluid (CSF) examination found that increased cell numbers of 51 cells raised $\mathrm{IgG}$ protein to $24.7 \mathrm{mg} / \mathrm{dL}$, and antibodies against the herpes simplex virus was positive. It was suggested that he should take a scalp video-EEG (high frequency filter at $70 \mathrm{~Hz}$, low frequency filter at 0.3 $\mathrm{Hz}$ ) and a 3.0T magnetic resonance image (MRI). However, after several examinations of the scalp video-EEG, his EEG showed a time-locked abnormal discharge synchronized to the rhythmic jerk (Figure 1), seen as a sharp-slow wave located in the right central-parietal lobe. The patient's MRI was normal. On the basis of the current clinical syndrome and the examination results, a diagnosis of encephalitis was made along with suspected EPC.

However, can the recent abnormal movement be interpreted as EPC? Based on the detailed functional anatomical analysis, we found that the location of the shoulder joint was close to the hip, which is connected to the trunk in the
Brodmann 4 area. Additionally, the rhythmic jerk could be described as a clonic seizure. After such analysis, we treated the patient with experimental antiepileptic medication, such as levetiracetam at $1.0 \mathrm{~g} /$ day. After 3 days, the abnormal rhythmic jerk of the shoulder-trunk-hip decreased and gradually disappeared once the dose was increased to $1.5 \mathrm{~g} /$ day. The combined functional anatomical analysis, the synchronized abnormal EEG, and clinical therapy allowed us to make a final diagnosis of EPC and encephalitis.

\section{Discussion}

EPC is a kind of clinical syndrome defined as continuous spontaneous jerking, confined to one part of the body, sometimes aggravated by action or sensory stimuli, occurring over hours, days, or even years without spreading to other body regions. Some authors have broadly defined EPC on clinical (semiological) grounds, whereas others have demanded additional electrophysiological evidence regarding the cortical origin of the phenomena to be considered. ${ }^{5}$ Typical clinical signs of EPC are: repetitive myoclonic jerks with hemiparesis or with other cortically-generated deficits; monomorphic, simple, brief excursions of the affected limb; regular or irregular occurrence of the jerks; and involvement of distal rather than proximal muscle groups. Additionally, electrophysiological studies of time-locked discharge can contribute to an enhanced degree of certainty. ${ }^{6}$ However, in some complex situations, clinical symptoms and detailed

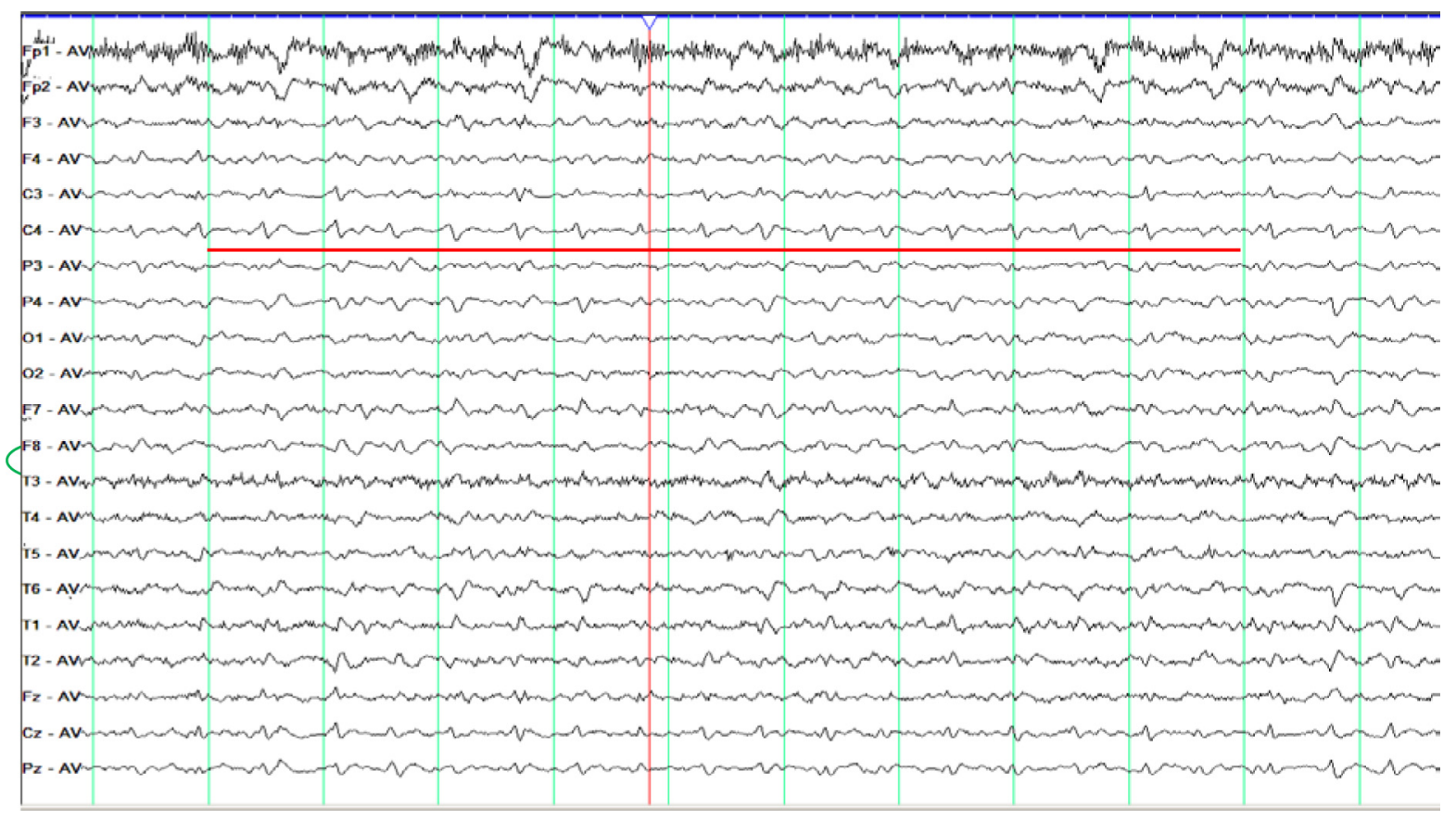

Figure I Abnormal discharge synchronized to the rhythmic jerk located in the right C4-P4.

Note: Red line indicates abnormal discharge synchronized to the rhythmic jerk located in the right C4. 


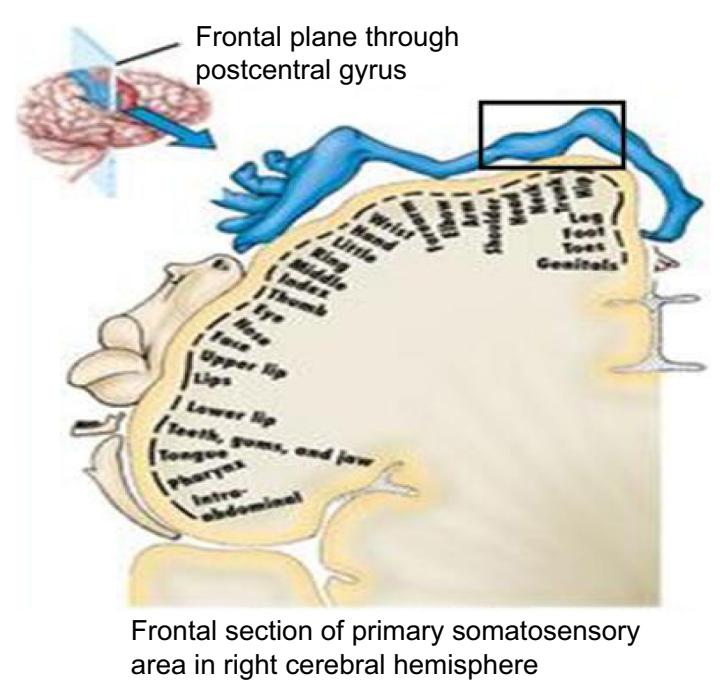

Figure 2 Shoulder joint-trunk-hip joint is located in a close area of Brodmann 4.

functional anatomical analysis of the Brodmann 4 area seem to be much more important to take into account as in our case. As shown in Figure 2, the shoulder, trunk, and hip are located in a certain area of Brodmann 4, epileptic discharges can lead to synchronized abnormal movement of shoulder jointtrunk-hip joint, and regular occurrence of the jerks can be interpreted as a clonic seizure. With antiepileptic treatment, the symptoms disappeared, and the seizures stopped.

EPC has been considered to originate from the cortex. However, in some special cases, scalp EEG cannot capture the discharge due to the smaller range of electrical activity, weaker epileptic potential, or a different position of the electrode. So, in such situations, functional anatomical analysis seems much more important. Reported elsewhere, other methods have been applied to confirm EPC, such as jerk-locked back-average analysis or functional MRI (fMRI), and so on. Espay et al reported an EPC case of hemifacial spasm which was diagnosed by applying the EEG-fMRI technique in $2008,{ }^{7}$ which may be our limitation.

Additionally, we should pay attention to the etiology of EPC. In 1993, Schomer ${ }^{8}$ reported that in childhood, the most frequent cause of EPC is Rasmussen encephalitis. At older ages, vascular and neoplastic diseases are the most frequent causes. Metabolic, toxic, and other immune-mediated causes account for most of the remaining cases. However, recently some other special EPC cases have been reported: Hemb et $\mathrm{al}^{9}$ presented an EPC case of dysplasia; Lv et al reported an EPC case accompanied by dystonia caused by Schizencephaly; ${ }^{10}$ Karkare et al showed an EPC case of mitochondrial encephalopathy; ${ }^{7} \mathrm{Li}$ et al presented an EPC case of Alpers-Huttenlocher syndrome; ${ }^{11}$ and Chhabra et al reported an EPC case of hypocalcemia. ${ }^{12}$ In this EPC case, positive antibodies against herpes simplex virus in the CSF was found. So, a detailed analysis of etiology also seems equally important. In addition, antiepileptic drugs or surgery may be the alternative choice according to the different etiology.

\section{Conclusion}

Our report expands the clinical presentation of EPC and provides the detailed anatomical and functional correlation to shoulder joint-trunk-hip joint abnormal movements. Although the abnormal movement presented in our case has not been described before, our case should call for the attention of clinicians to the potential epileptic seizure.

\section{Acknowledgment}

The authors thank Dr Jiang Wu who was involved in conceiving the manuscript and revising it critically for important intellectual content.

\section{Author contributions}

YDL participated in the drafting of the manuscript. WZ collected the clinical data. FNC helped to draft the manuscript. CL participated in its design and coordination. HMM conceived the case and is accountable for the integrity of any part of the work. All authors contributed toward data analysis, drafting and revising the paper and agree to be accountable for all aspects of the work.

\section{Disclosure}

The authors report no conflicts of interest in this work.

\section{References}

1. Obeso JA, Rothwell JC, Marsden CD. The spectrum of cortical myoclonus. From focal reflex jerks to spontaneous motor epilepsy. Brain. 1985;108(Pt 1):193-224.

2. Thomas JE, Reagan TJ, Klass DW. Epilepsia partialis continua. A review of 32 cases. Arch Neurol. 1977;34(5):266-275.

3. Vukadinovic Z, Hole MK, Markand ON, Matt BH, Sokol DK. Lingual epilepsia partialis continua in a girl. Epileptic Disord. 2007;9(3): 323-326.

4. Espay AJ, Schmithorst VJ, Szaflarski JP. Chronic isolated hemifacial spasm as a manifestation of epilepsia partialis continua. Epilepsy Behav. 2008;12(2):332-336

5. Cockerell OC, Rothwell J, Thompson PD, Marsden CD, Shorvon SD Clinical and physiological features of epilepsia partialis continua: Cases ascertained in the UK. Brain. 1996;119(Pt 2):393-407.

6. Wieser HG, Graf HP, Bernoulli C, et al. Quantitative analysis of intracerebral recordings in epilepsia partialis continua. Electroencephalogr Clin Neurophysiol. 1978;44:14-22.

7. Karkare K, Sinha S, Ravishankar S, et al. Epilepsia partialis continua in mitochondrial dysfunction: Interesting phenotypic and MRI observations. Ann Indian Acad Neurol. 2008;11(3):193-196.

8. Schomer DL. Focal status epilepticus and epilepsia partialis continua in adults and children. Epilepsia. 1993;34 Suppl 1:S29-S36. 
9. Hemb M, Paglioli E, Dubeau F, et al. "Mirror EPC": epilepsia partialis continua shifting sides after rolantic resection in dysplasia. Neurology. 2014;83(16):1439-1443.

10. Lv Y, Ma D, Meng H, Zan W, Li C. A case of schizencephaly has a normal surface EEG but abnormal intracranial EEG: epilepsia partialis continua or dystonia? Clin EEG Neurosci. 2013;44(4): 319-323.
11. Li S, Guo J, Ying Z, et al. Valproic acid-induced hepatotoxicity in Alpers syndrome is associated with mitochondrial permeability transition pore opening-dependent apoptotic sensitivity in an induced pluripotent stem cell model. Hepatology. 2015;61(5):1730-1739.

12. Chhabra P, Modi M, Garg A, Prabhakar S, Bhadada SK. Hypocalcemia: A rare cause of epilepsia partialis continua. Neurol India. 2012;60(4): 432-433.

\section{Publish your work in this journal}

Neuropsychiatric Disease and Treatment is an international, peerreviewed journal of clinical therapeutics and pharmacology focusing on concise rapid reporting of clinical or pre-clinical studies on a range of neuropsychiatric and neurological disorders. This journal is indexed on PubMed Central, the 'PsycINFO' database and CAS, and is the official journal of The International Neuropsychiatric Association (INA). The manuscript management system is completely online and includes a very quick and fair peer-review system, which is all easy to use. Visit http://www.dovepress.com/testimonials.php to read real quotes from published authors.

Submit your manuscript here: http://www.dovepress.com/neuropsychiatric-disease-and-treatment-journal 